\title{
Philosophiques
}

\section{Marie-Michèle Blondin, Vivre et vivre encore : la notion de vie chez Arthur Schopenhauer, Hildesheim, Georg Olms, coll. Europaea Memoria, 2019, 278 pages}

\section{Ugo Batini}

Volume 47, numéro 2, automne 2020

URI : https://id.erudit.org/iderudit/1075141ar

DOI : https://doi.org/10.7202/1075141ar

Aller au sommaire du numéro

Éditeur(s)

Société de philosophie du Québec

ISSN

0316-2923 (imprimé)

1492-1391 (numérique)

Découvrir la revue

Citer ce compte rendu

Batini, U. (2020). Compte rendu de [Marie-Michèle Blondin, Vivre et vivre encore : la notion de vie chez Arthur Schopenhauer, Hildesheim, Georg Olms, coll. Europaea Memoria, 2019, 278 pages]. Philosophiques, 47(2), 516-521. https://doi.org/10.7202/1075141ar d'utilisation que vous pouvez consulter en ligne.

https://apropos.erudit.org/fr/usagers/politique-dutilisation/ 
Puisque la musique, à laquelle Thomas Dommange a consacré notamment un autre très beau livre ${ }^{5}$, nous fait précisément entrer dans un monde débarrassé de la causalité, et qu'elle est un langage réfractaire à la signification, l'être singulier tient, selon l'auteur, un discours «musical» — et «musicales» sont par nature les figures telles qu'il les a définies. La musique apparaît encore dans Le rapt ontologique à un autre propos, lorsque Dommange fait un rapprochement entre la forme dramatique et une ligne mélodique. Par exemple, peut-être à la manière de cette petite phrase musicale par laquelle Wittgenstein aurait aimé cristalliser sa vie, l'Hymne à la joie de la Neuvième Symphonie de Beethoven n'est pas le centre ou le sommet de l'œuvre, mais sa réalité première, son principe signifiant, l'équivalent d'un concept - horizon du premier temps de la double ontologie prônée par Dommange.

Au terme de ce livre nous apparaît le vrai visage de la singularité : distincte de l'individualité, n'appartenant pas en propre aux humains, elle consiste en la figure naissant de l'empire qu'une idée a sur un être, figure sur laquelle nous n'avons pas plus de prise qu'un animal sur sa parure. Très original par ses thèses et par les parentés qu'il décèle ainsi entre des réalités qui, à première vue, nous sembleraient étrangères les unes aux autres, Le rapt ontologique est une œuvre philosophique profondément travaillée par la poésie - comme l'auront probablement suggéré quelques-unes des citations qui précèdent. Il saura plaire à tous ceux qui ont à cœur d'échapper au cloisonnement des disciplines, et qui recherchent l'alliance de la pensée et de l'imagination.

THIERRY LAISNEY

Université Paris Diderot

\begin{abstract}
Marie-Michèle Blondin, Vivre et vivre encore: la notion de vie chez Arthur Schopenhauer, Hildesheim, Georg Olms, coll. Europaea Memoria, 2019, 278 pages.
\end{abstract}

Issu d'un travail de doctorat soutenu en 2015, Vivre et vivre encore de Marie-Michèle Blondin se propose d'étudier la notion de vie au sein de la philosophie de Schopenhauer. Cet ouvrage s'attache à montrer comment, au sein d'une telle métaphysique, la volonté explique et justifie le phénomène de la vie, mais aussi sa persistance et son affirmation à travers ce que M.-M. Blondin appelle le "vivre-encore». En prenant comme point de départ une définition métaphysique de la vie fondée sur le concept schopenhauerien de "volonté de vivre», l'auteure arrive à articuler les différentes

5. Thomas Dommange, L'homme musical. La notation en mots dans l'œuvre de Schumann, Besançon, Les Solitaires Intempestifs, 2010. 
approches de la vie - biologique, philosophique, existentielle, voire métaphorique - mais engage surtout une remise en question de la solution que Schopenhauer lui-même propose au problème de l'existence à travers une critique du salut que constitue la négation de la volonté. Si ce dernier point est tout à fait cohérent avec l'ensemble de l'ouvrage, il ne manque pas néanmoins de poser certains problèmes.

Avec raison, M.-M. Blondin commence par un constat simple: l'attention quasi exclusive portée par les commentateurs à la question de la souffrance a fini par faire écran à une réflexion complète sur la vie. Il s'agit donc pour elle d'engager son étude en saisissant la vie à partir du sentiment de vie et de rendre ainsi plus compréhensible l'attachement presque viscéral de chacun à son existence, et cela malgré la souffrance. La vie et le vivre-encore ne résulteraient pas d'un choix, mais de la nature même de notre propre essence. "L'hypothèse défendue est que le phénomène de la vie contient toutes les raisons pour vivre et vivre encore» (p. 26); il s'agira alors d'explorer au maximum cette hypothèse en étudiant successivement quatre aspects de la vie: sa définition, son sentiment, sa connaissance et sa persistance. Pour ce faire, l'ouvrage procède de façon progressive en explicitant dans les chapitres I et II la définition de la vie chez Schopenhauer, puis en évoquant au chapitre III la question du sentiment afin d'entrer au cœur des difficultés en travaillant le double sens de l'affirmation de la volonté au chapitre IV, et la question de la négation de la volonté comme antinature au chapitre V, avant de conclure au chapitre VI par une tentative de dépasser la voie tracée par Schopenhauer en montrant comment la vie peut être moins douloureuse que le philosophe ne le prétend.

Le chapitre premier s'ouvre sur la difficulté classique qu'il y a à définir le vivant et montre comment Schopenhauer arrive à surmonter celle-ci en accédant à une connaissance immédiate et intime de soi qui conduit le philosophe à identifier sa propre essence à la volonté. "La vie définie comme "manifestation de la volonté" doit être considérée comme la définition métaphysique de la vie chez Schopenhauer" (p. 37). La genèse de cette idée tient tout d'abord au constat que la vie n'est que souffrance et misère. Mais Schopenhauer envisage aussi la vie d'un point de vue biologique et athée, ce qui l'amène à croiser les physiologistes français ainsi que la pensée de Goethe, qui a un impact considérable sur le développement de la sienne, mais aussi celle de Schelling, même si Schopenhauer cherche toujours à atténuer son influence. L'enjeu décisif de ce chapitre est de montrer que si la vie se définit comme volonté, la réciproque n'est pas pour autant valable. Le paragraphe $54 \mathrm{du}$ Monde, central pour cette étude, souligne bien que «ce que veut la volonté est toujours la vie ${ }^{1}$, mais cela ne signifie pas pour autant que la première se réduit à la seconde. Il est donc nécessaire pour

1. Le monde comme volonté et comme représentation, Paris, Gallimard, «Folio essais ", 2009, $\$ 54$, p. 530. 
saisir dans toute sa complexité l'idée de vie de ne pas se limiter au point de vue subjectif que le sujet a sur elle et d'adopter aussi un point de vue objectif qui correspond en grande partie à celui des sciences de la nature - point de vue amplement développé d'ailleurs dans un ouvrage complet, De la volonté dans la nature (1836). Il n'y a qu'ainsi que l'on pourra comprendre le caractère métaphysique d'essence du monde propre à la volonté. M.-M. Blondin appuie cette réflexion sur une traduction d'un passage du Nachlass (dont on retrouve en annexe de l'ouvrage quelques extraits): "La volonté n'est pas, comme on l'a supposé jusqu'à présent, un phénomène de la vie seule, mais plutôt c'est la vie qui est le phénomène de la volonté ${ }^{2}$ (p. 57).

Le chapitre II prend acte de cette duplicité de la vie qui est pour l'homme à la fois vécue (point de vue subjectif) et connue (point de vue objectif), et aborde les différents "visages" que la notion prend au sein de l'œuvre de Schopenhauer. Ce chapitre est stimulant, même si l'on peut regretter que l'étude de la dimension biologique ne soit pas l'occasion de revenir plus en détail sur les sources physiologiques de Schopenhauer, et tout particulièrement sur l'influence de son maître à l'Université de Göttingen, J.-F. Blumenbach, qui a promu une dynamique nouvelle du vivant avec son concept de «force formatrice» discuté par Kant, Schelling et Goethe, c'està-dire précisément par les sources mises en avant dans le chapitre précédent. Le passage sur la définition philosophique de la vie est important, car il permet de préparer la discussion qui aura lieu dans les chapitres suivants au sujet de la négation ou de l'affirmation de la volonté. En effet, aborder la vie en philosophe revient à la considérer in abstracto et donc à percevoir celle-ci de manière complète.

Les chapitres III, IV et V constituent, selon nous, le cœur problématique de l'ouvrage. Ils discutent l'alternative entre l'affirmation et la négation de la volonté une fois que cette dernière est parvenue à s'appréhender elle-même. En se focalisant sur le sentiment et la connaissance de la vie, le chapitre III remet en perspective la question de la souffrance. L'auteure rappelle fort opportunément que ce qui est premier, ce n'est pas la souffrance, mais bien la volonté, ainsi ce sentiment de vie ne doit pas exister, selon elle, seulement comme souffrance. M.-M. Blondin déconstruit minutieusement cette identification, même si le fait que la volonté soit première n'empêche nullement que la vie soit toujours perçue, en vertu de sa nature d'ailleurs, comme souffrance par le sujet vivant. Le texte ne cherche pas à esquiver la difficulté et est souvent justement éclairé par des notes qui interrogent objectivement le problème, comme par exemple la note 5 qui reconnaît: «Il est vrai, par ailleurs, que dans les faits la souffrance est une manifestation simultanée à celle de la volonté » (p. 127). L'objectif principal est de montrer que dans ce sentiment de vie se manifeste aussi un élan, une poussée vers la vie - mais une fois encore cette tendance n'exclut en aucun cas la possibilité 
d'une souffrance. Avec justesse, le chapitre rappelle la place centrale, car principielle du point de vue de la connaissance, du sentiment, replaçant une fois de plus le corps au centre du questionnement (avec une analyse stimulante de l'opposition du cœur et de la tête). C'est bien par la connaissance du sens interne et donc du corps-propre que Schopenhauer accède au secret du monde. Cela met en avant quelque chose d'essentiel pour la suite: "Ce n'est ni dans la raison ni de l'extérieur que la vie trouve les raisons de son existence et de sa persistance, mais bien dans le cœur même de l'individu » (p. 153).

Le chapitre IV prolonge cette analyse en considérant ce qu'il nomme la vie vivante, c'est-à-dire une vie qui, de part en part, est affirmation de la volonté. Un des grands mérites de l'ouvrage consiste à livrer une étude précise de cette affirmation alors que la plupart des commentateurs ont eu tendance à délaisser ce mouvement essentiel de l'œuvre au profit de la seule négation. Reconduisant l'analyse de Vincent Stanek ${ }^{3}$, l'auteure insiste bien sur les deux sens de cette affirmation: celle-ci se comprend tout d'abord comme un effort aveugle vers la manifestation et peut donc être perçue comme le principe même de l'objectivation de la volonté, mais elle prend aussi un sens plus élevé, lié à la prise de conscience de la volonté par ellemême. On peut voir alors dans cette seconde affirmation une affirmation de la vie. Tout cela prépare évidemment en retour une compréhension de la négation comme une «vie atrophiée» (p. 168) voire niée, c'est-à-dire le choix de la mort contre celui de la vie.

Avec cette distinction entre une "vie vivante», une "vie atrophiée» et une "vie niée ", nous entrons au cœur de toutes les tensions et nous retrouvons aussi le moteur principal du livre IV du Monde qui identifie comme «le grand problème» le fait de vouloir ou ne pas vouloir la vie. Le chapitre $\mathrm{V}$ expose les tenants et les aboutissants de la négation de la volonté, n'hésitant pas à voir en elle un choix «funeste» (p. 164, 171, 179). En effet, si une souffrance extrême peut engendrer subitement la négation de la volonté, en règle générale, cette négation est liée à une prise de conscience qui deviendra aux yeux de Schopenhauer une forme de salut par la connaissance. Cependant, il ne faut pas imaginer que ce renversement de notre propre essence soit aisé et consiste en un simple renoncement. Il est une lutte acharnée avec ce qui constitue le plus en propre notre nature - un tel combat ne peut donc être lui aussi qu'accompagné de souffrance. M.-M. Blondin affirme alors clairement sa position: "La négation de la volonté est une fausse solution [...] [il s'agira alors de] remettre en doute la possibilité même de cette solution" (p. 180). C'est donc bien une thèse forte qu'entend défendre cet ouvrage dans ses deux derniers chapitres.

La théorie du salut qu'élabore Schopenhauer est impossible à accepter pour ceux qui souhaitent «vivre». Si la solution semble bancale, c'est que ce 
que l'individu gagne en niant la volonté, il le perd en même temps que sa vie dans la négation totale de la volonté. Cette remarque est centrale mais s'attache trop, selon nous, au point de vue individuel — point de vue qu'il s'agit d'ailleurs de dépasser dans la négation qui commence précisément par une négation du principe d'individuation. On touche alors là un des points les plus sensibles de l'argumentation, une difficulté qui court depuis le début de l'ouvrage et qui est liée au statut du plaisir. Car si l'auteure n'hésite pas à avancer au chapitre IV que "[c]ette vie [la vie vivante] souffre, pas nécessairement ou absolument toutefois, car elle s'octroie des plaisirs et des jouissances» (p. 156), elle trouve problématique "que celui qui nie la volonté se trouve empli de joie intérieure et de félicité véritable» (p. 181). Et on la comprend, car cette «jouissance» repose sur un «ne plus sentir». Mais cette difficulté est liée à la compréhension même de la nature des plaisirs. Or ceux-ci, comme le rappelle Clément Rosset ${ }^{4}$, ne sont pas problématiques chez Schopenhauer parce qu'ils seraient moins nombreux au cours d'une vie mais parce qu'ils sont moins réels. Il aurait donc fallu construire, bien avant le chapitre VI, une véritable ontologie du plaisir pour comprendre mieux les enjeux des questions centrales que soulève cet ouvrage. Si Schopenhauer juge incertains les plaisirs de la vie, il a recours néanmoins aux faits à la fin du Monde pour illustrer la jouissance secrète que porte avec elle cette victoire de la volonté sur elle-même. C'est le rôle même des références à la mystique. La figure du saint est d'ailleurs, comme le révèle Nietzsche ${ }^{5}$ luimême, un point central de la doctrine et constitue une forme de figuration de cette joie étonnante dont les tableaux du Corrège ou de Raphaël peuvent nous donner un faible aperçu. En se tournant résolument du côté de la vie, l'ouvrage ne prend pas assez en considération les conditions et les conséquences de la voie de la négation qui repose principalement sur toute une littérature mystique qui constitue, avec la physiologie, une autre des grandes sources de la métaphysique schopenhauerienne. Or le sourire des saints, comme celui de Bouddha qui fascine tant Schopenhauer, soutient bien une forme de positivité au sein de cette négation. De même, la douleur propre à cette lutte contre soi-même ne peut pas se penser sur le même plan que la souffrance inhérente à la vie car elle se trouve dotée, sur la voie de la négation, d'un sens qui manque cruellement aux douleurs spécifiques à la vie.

Le dernier chapitre entend dépasser cette «fausse solution» (p. 200) que représente la voie de la négation en revenant sur le vivre-encore et en montrant à quel point cet attachement à la vie émane du plus profond de notre être. Ce dernier est si puissant que Schopenhauer lui-même, conscient de la difficulté propre au salut, n'hésite pas dans les Parerga à constituer une eudémonologie, c'est-à-dire à rechercher une forme d'accommodement avec

4. Clément Rosset, Schopenhauer, philosophe de l'absurde, Paris, PUF, 1989, p. 65.

5. Friedrich Nietzsche, Par-delà bien et mal, Paris, Gallimard, "Folio essais", 1971, \$ 47, p. 65. 
la vie fondée sur une affirmation tempérée de la volonté. Mais pour celui qui veut vivre pleinement, la vie "sage" peut finalement paraître pire que la vie de souffrance. Et cela car, l'auteure le souligne à nouveau, il ne faut pas oublier - même si Schopenhauer n'en fait pas la publicité — qu'il y a dans la vie aussi des moments d'accalmie. En effet, la vie offre bien par moments de la "jouissance» (rôle central du rapport sexuel), de la gaieté, et grâce à l'expérience esthétique parfois un repos temporaire. L'évocation de cette dernière constitue ici à nos yeux un dernier point de tension. En effet, par bien des aspects, la contemplation esthétique peut se comprendre dans le voisinage de l'expérience mystique et donc être considérée comme une sorte de préambule à la négation. Cela est d'autant plus vrai que la désindividualisation qu'elle provoque fait partie du même régime de plaisir que celui qui est propre à la négation et devrait donc être aussi problématique aux yeux de l'auteure. Comment, tout simplement, l'expérience esthétique peut-elle prendre place au sein du sentiment de vie du sujet? Cette difficulté est en partie discutée par M.-M. Blondin qui constate que ce «vrai bonheur» ne résulte "pas d'une intensification du sentiment de vie et des forces vitales" (p. 232). Cette jouissance en dehors du corps et donc en un sens par-delà la vie ne peut manquer d'interroger.

Toutes ces questions qui demeurent à la fin de l'ouvrage montrent à quel point ce dernier peut être stimulant, et cela car il défend jusqu'au bout une thèse originale qui a le mérite d'interroger en profondeur certains dogmes de la pensée schopenhauerienne.

UGO BATINI

Université de Poitiers

Juan Vincente Cortés, La notion de jouissance chez Spinoza. Essai de reconstruction conceptuelle, Paris, Éditions de la Sorbonne, coll. «La philosophie à l'œuvre», 2019, 172 pages.

"Toujours du sentiment! comme si le monde ne contenait pas autre chose ${ }^{1}$.» Cette citation de Flaubert, mise en exergue du présent ouvrage, donne le ton de la réflexion menée par Juan Vincente Cortés. Docteur en philosophie et chercheur adjoint à l'Universidad Alberto Hurtado à Santiago du Chili, il enseigne actuellement la philosophie moderne. En publiant la première partie de sa thèse (préparée sous la direction de Chantal Jaquet et soutenue à l'Université Paris-I Panthéon Sorbonne en 2014), il a pris le risque de se joindre à la longue liste des commentateurs de Spinoza, qui, depuis de nombreuses années, tentent de rendre toujours plus intelligible sa philosophie,

1. Gustave Flaubert, Bouvard et Pécuchet. Cette citation fait partie de celles que l'auteur a placées en exergue de son livre. 